\title{
Chitosan-Reinforced MFC/NFC Aerogel and Antibacterial Property
}

\author{
Huanlei Yang, Shuxin Zhang, and Jinhua Yan $(D)$ \\ Guangdong Industry Polytechnic, Guangzhou 510300, China \\ Correspondence should be addressed to Jinhua Yan; jhyan2013@163.com
}

Received 31 May 2019; Revised 18 October 2019; Accepted 28 October 2019; Published 26 August 2020

Academic Editor: Mingzhi Huang

Copyright (C) 2020 Huanlei Yang et al. This is an open access article distributed under the Creative Commons Attribution License, which permits unrestricted use, distribution, and reproduction in any medium, provided the original work is properly cited.

\begin{abstract}
MFC/NFC aerogel has water sensitivity, and it should be improved in strength in water before application. Chitosan was investigated as a MFC/NFC aerogel reinforcing agent in this paper. The reinforced aerogel showed slightly tighter structure and very good water stability and mechanical strength. FTIR disclosed the chemical bonds formed between chitosan and cellulose. Nanoparticles of silver (Ag-NPs) were loaded using the reinforced aerogel. The excellent Ag-NP monodistribution on the aerogel was expressed by TEM. Both chitosan-reinforced Ag-NPs loaded MFC aerogel and NFC aerogel and expressed great antibacterial activity, though reinforced MFC aerogel exhibited better properties, like higher BET, lighter density, more Ag-NP loading, and better distribution, than NFC aerogel in this research. Chitosan-reinforced MFC aerogel is a good potential substrate for nanoparticle loading and biocomposite making.
\end{abstract}

\section{Introduction}

Aerogels are porous materials of interconnected nanostructures made from gels by replacing the liquid by gas, which exhibit unusual properties, such as high porosity and surface area, low density, and low heat conductivity [1]. A wide variety of aerogels have been reported in the literature. They can be produced from silica, alumina, tin oxide, chromia, and carbon, with silica being the most widely used one [2]. The unique properties associated with aerogels have led to their use in a wide range of applications such as catalysts [2], catalyst supports [3], superthermal and sound insulators [2,4], electronics, particle filters, and as a storage media for gases in fuel cells [5, 6]. More recently, cellulose has gained interest as a source material for the production of aerogels due to its renewability and biodegradability [7]. Cellulose aerogels have proven particularly useful in applications where biocompatibility and biodegradability are needed, such as for medicinal, cosmetic, and pharmaceutical applications, opening up the application fields of aerogels even wider [8]. Micro/nanofibrillar cellulose (MFC/NFC) is isolated from natural cellulose fibers by basically mechanical action after enzyme or chemical pretreatment. Its higher aspect ratio made $\mathrm{MFC} / \mathrm{NFC}$ an attractive application of providing a more beneficial template to accommodate nanoparticles, for example, NFC with silver nanoparticles using UV reduction [9], NFC with magnetic particles [10], and NFC with drug nanoparticles [11].

MFC/NFC aerogels generally can be prepared by two steps: one is the MFC/NFC suspension generated by a unique method of enzymatic/chemical pretreatment and mechanical fibrillation by a microfluidizer and the other is to produce aerogel by freezing the suspensions and dried in a special way [7, 12-15]. Different cellulose sources and preparation processes make the MFC/NFC cellulose aerogels with totally different microstructures and properties $[16,17]$. MFC/NFC has been widely investigated recently due to its sustainable, renewable, and biocompatible nontoxic properties for relatively high physical-chemical stability materials $[18,19]$. The density and surface texture of the aerogels can be tuned by selecting the concentration of the MFC/NFC dispersion before freeze-drying. All of these aerogels are based on the hydrogen bond formation between MFC/NFCs [20]. Unfortunately, these hydrogen bonds can be easily destroyed by immersing the aerogels in water, which limited the application of the aerogels [20]. 
Chemical cross-linking affords a method for the preparation of MFC/NFC cellulose aerogels with high mechanical properties [21-23]. Although these efforts have been carried out for the improvement of the mechanical properties of cellulose aerogels, the substances that can provide safe, renewable, and biocompatible nontoxic properties are more desirable. Chitosan from the natural polymer chitin has good biocompatibility, as well as extensive applications in pharmacology, biomedicine, agriculture, food, and waste treatment [24]. Regarding the chemical nature, chitosan and cellulose have similar structures, with the same $\beta$-glycosidic linkages, being the main difference as the presence of primary amino groups at most of the C-2 positions in chitasan, instead of the hydroxyl groups. Much work has been devoted to the study of chitosan blends with cellulose [25-28], poly(vinyl alcohol) [29-31], gelatin [32], collagen [33], and others [34]. The evidence of some chitosan-cellulose chemical interactions was proved [19]. Our group also reported that chitosan improved the nanopaper mechanical strength both in dry and wet states by cross-linking [35]. That is, chitosan can be used as a cross-linker of cellulose and nanofibrils.

In the current work, we firstly reported chitosan-reinforced MFC/NFC aerogels and their application. The aerogels showed improved mechanical properties. Using the reinforced aerogel as a template, nanoparticles, silver as an example, were loaded uniformly.

\section{Materials and Methods}

2.1. MFC/NFC Preparation. Fully bleached eucalyptus pulp was passed through the microgrinder, 6 passes, at a concentration of $2.31 \%$, to produce MFC suspension. Then some of the MFC suspension was treated by TEMPO before passing the microfluidizer 10 times to produce NFC. The TEMPO oxidation technique was performed as given in the literature with sodium hypochlorite as the terminal oxidant [36]. Dialysis was applied to remove extra salts in NFC using Nomial MWCO 3500 (Fisherbrand regenerated cellulose) for $48 \mathrm{~h}$ with Milli-Q water changing frequency every $5 \mathrm{~h}$. The acidic group content of MFC and NFC was measured as 70 and $400 \mu \mathrm{mol} / \mathrm{g}$, respectively, by titration as per the literature [37]. The titration steps were as follows: $0.01 \mathrm{M}$ $\mathrm{NaOH}$ was added drop by drop till there was no further drop in system conductivity. Then the amount of $\mathrm{NaOH}$ was recorded till the conductivity increased. The consumed $\mathrm{NaOH}$ can be calculated to get the acidic group content. Both MFC and NFC ready-to-use slurries were kept in a refridgerator.

2.2. Chitosan Solution Preparation. Chitosan (MW 10253, viscosity $20 \mathrm{cP}$ ) was purchased from Aldrich (degree of deacetylation: $\sim 85 \%$ ). Chitosan solutions were prepared by dissolving chitosan ( $2 \%$ by weight) in ( $1 \%$ by volume) aqueous acetic acid solution [38]. The solution was sonicated for $30 \mathrm{~min}$ before use.
2.3. MFC/NFC Aerogel Preparation. MFC/NFC dispersion with chitosan mixture at different ratios of 100/0, 90/10, 80/ 20 , and 70/30 (MFC to chitosan weight) was mixed to $0.5 \%$ by adding DI water, by using a high-shear mixer for $5 \mathrm{~min}$, followed by a magnetic bar stirring for $1 \mathrm{~h}$, and then sonication for another $30 \mathrm{~min}$. Then the mixture was centrifuged to remove water at a speed of $1.2 \times 104 \mathrm{rpm}$ for $1 \mathrm{~h}$. A gel was formed. The gel was frozen by liquid nitrogen, then placed on a freeze dryer (Labconco) for at least $48 \mathrm{~h}$. The properties of density, strength, and BET surface area were examined. The density was measured by the volume and weight of each aerogel. The strength was evaluated by its water stability which was conducted in water for stirring at a given time. After Ag-NPs loaded, both the MFC/CH aerogel and NFC/ $\mathrm{CH}$ aerogel BET surface areas were measured after $4 \mathrm{~h}$ degassing at $105^{\circ} \mathrm{C}$ (Gemini VII Series surface area analyzer, Micromeritics Instrument Corporation).

2.4. Nanoparticle Loading. The in situ loading of silver nanoparticles (Ag-NPs) onto the aerogels was carried out through the reduction of $10 \mathrm{mM} \mathrm{AgNO}_{3}$ solution. Under ambient temperature $\left(\sim 25^{\circ} \mathrm{C}\right), \mathrm{AgNO}_{3}$ solution was absorbed by the porous matrix by soaking or dropwise addition, keeping totally wet for enough time till the aerogels are not absorbing solution anymore. After a certain time of air drying, a partially dehydrated matrix was obtained. It was then immersed in an aqueous solution of $\mathrm{NaBH}_{4}(50 \mathrm{mM})$ for $20 \mathrm{~min}$. The color of the samples turned to yellow or dark brown due to the reduction of $\mathrm{Ag}+$ into silver nanoparticles. The composite was rinsed with Milli-Q water three times to remove water-soluble substances and unbound silver particles. Finally, the composite was freeze dried.

2.5. Aerogel Morphology. SEM sample preparation of the MFC/NFC slurry and aerogels with/without Ag-NPs:MFC/ NFC $(20 \mathrm{~mL}, 0.08 \%)$ was taken for magnetic stirring for $45 \mathrm{~min}$, followed by $30 \mathrm{~s}$ sonication. A silicon plate was used as the MFC/NFC material support. Firstly, the plate was cut into small pieces, which were soaked in $10 \% \mathrm{NaOH}$ solution for $30 \mathrm{~s}$ and then in Milli-Q water. These silicon pieces were dried by nitrogen blowing, followed by UV exposure for 20 min. A tiny drop of the abovementioned MFC/NFC slurry was placed on the surface of a cleaned silicon piece. Overnight air drying was needed before the SEM test. The samples were coated with gold before SEM operation. The porous structures of MFC/NFC aerogels were examined using a field emission scanning electron microscope (FESEM) at an accelerating voltage of $20 \mathrm{kV}$ (a high resolution JEOL 6400F cold field emission SEM). The MFC/NFC aerogels with Ag-NPs were characterized using VPSEM (Variable Pressure Scanning Electron Microscope, Hitachi S3200N) with an energy dispersive X-ray spectrometer).

TEM preparation of MFC/NFC aerogels with Ag-NPs: A JEOL 2000FX transmission electron microscope (TEM) operating at $20.0 \mathrm{kV}$ was utilized to define the $\mathrm{Ag}-\mathrm{NPs}$ in the MFC/NFC aerogels. A specimen can be prepared by cutting the sample into thin slices using a diamond saw, then cutting 3-mm-diameter disks from the slice, thinning the disk on a 
grinding wheel, dimpling the thinned disk, and then ion milling it to electron transparency.

2.6. FTIR Spectroscopy. FTIR spectroscopy was performed on a PerkinElmer Spectrum (Version 10.03.09). Spectra were obtained after the accumulation of 6 scans which had a resolution of $4 \mathrm{~cm}^{-1}$ over the range of $4,000-650 \mathrm{~cm}^{-1}$.

2.7. Thermal Gravimetric Analysis (TGA). In order to determine the thermal decomposition temperature of composite aerogels, thermogravimetric analysis (TGA) was used. It was operated on Perkin Elmer TGA Q500 with a heating rate of $10^{\circ} \mathrm{C} / \mathrm{min}$ to $500^{\circ} \mathrm{C}$ in a nitrogen atmosphere. In order to obtain the amount of Ag-NPs in aerogel, TGA under oxygen in the range of $500-575^{\circ} \mathrm{C}$ was continued. The isothermal time for $25 \mathrm{~min}$ at $575^{\circ} \mathrm{C}$ was utilized.

2.8. Antibacterial Activity Test. The antibacterial activity of the aerogels was tested against Escherichia coli (E. coli), Gram-negative bacteria, using the viable cell-counting method. Briefly, about $100 \mu \mathrm{LE}$. coli was cultivated in $100 \mathrm{~mL}$ of a nutrient broth solution to give a bacterial concentration of about $7 \times 1011 \mathrm{CFU} / \mathrm{mL}$. Then, $1 \mathrm{~mL}$ of the bacteria/nutrient solution was added to $9 \mathrm{~mL}$ of sterilized nutrient broth solution $(0.8 \%)$. Several decimal dilutions were performed until the bacterial concentration increased from $7 \times 103$ to $7 \times 107 \mathrm{CFU} / \mathrm{mL}$. NFC, NFC/chitosan, and $\mathrm{MFC} /$ chitosan and their silver-loaded counterparts (NFC/ chitosan/Ag-NPs and MFC/chitosan/Ag-NPs) were used in the antibacterial tests. Accurate weight of the aerogel samples $(100 \mathrm{mg})$ was assigned to the experiment. To perform the antibacterial testing, aerogels of the respective samples were put into $10 \mathrm{~mL}$ of the bacteria/nutrient solution and incubated in a shaker at $37^{\circ} \mathrm{C}$ for $12 \mathrm{~h}$. After the exposure of the bacteria to films, $100 \mu \mathrm{L}$ of the bacterial solution was taken out and quickly spread on a plate containing nutrient agar. Plates containing bacteria were incubated at $37^{\circ} \mathrm{C}$ for $24 \mathrm{~h}$, and then the numbers of the surviving colonies were observed. These results were compared to the number of bacterial colonies of the untreated control.

\section{Results and Discussion}

3.1. MFC/NFC Aerogel Physical Properties. MFC/NFC aerogels with chitosan addition were examined for their densities and strength. The dry aerogel-retained stability in water is shown in Figure 1. MFC aerogels with $0 \%$ chitosan (A), $10 \%$ chitosan (B), and $20 \%$ chitosan (C) were soaked into water and kept under magnetic stirring at $610 \mathrm{rpm}$ for $4 \mathrm{~h}$. MFC aerogel with $0 \%$ chitosan was totally dispersed into water again, chitosan-added MFC aerogels were kept intact. In addition, a 10\% chitosan-added MFC aerogel compression test was conducted for mechanical strength property of $0.36 \mathrm{MPa}$. The dry aerogel density was tested by measuring the volume of the aerogel and its weight. Table 1 is the density calculation data of the produced aerogels under $2 \%$ concentration, which is in accordance with that of cellulosebased chitosan aerogel [25]. The data showed that, with increase in chitosan level, the aerogel density rose. It implied that chitosan can shrink the aerogel pores and tighten the MFC/NFC aerogel structure.

After Ag-NPs loaded, both MFC/CH aerogel and NFC/ $\mathrm{CH}$ aerogel surface areas were measured after $4 \mathrm{~h}$ degassing at $105^{\circ} \mathrm{C}$. The results are listed in Table 2. In Table 2, NFC/CH aerogel has lower BET data than $\mathrm{MFC} / \mathrm{CH}$ after $\mathrm{Ag}$ loaded. Due oxidation, the NFC possessed the acidic group content up to $400 \mu \mathrm{mol} / \mathrm{g}$, while MFC possessed the acidic group content of $70 \mu \mathrm{mol} / \mathrm{g}$. As a result, NFC was more active with chitosan and formed a tighter aerogel structure [25]. Accordingly, its BET is smaller than that of $\mathrm{MFC} / \mathrm{CH}$ aerogel. The higher BET data also imply that the aerogel has more space exposure to which gives pores benefit particles/solution filling. As a result, we found that the $\mathrm{MFC} / \mathrm{CH}$ aerogel loaded more nanoparticles than the NFC/CH aerogel (see Table 3).

3.2. MFC/NFC Aerogel Microscopy. MFC/NFC aerogels with different chitosan addition levels were conducted and scanned using a SEM. Generally, chitosan can cause the aerogel dense structure with the increase in addition level (see Figure 1). The structural change is clear also in these SEM images. When MFC aerogel contains 0\% chitosan, its structure is open and the pores are easily seen (Figure 1(a)). When a small amount of chitosan solution added (Figure 1(b)), we can see the light "films" formed among pores like fogs. With increasing chitosan content to $20 \%$ (Figure 1(c)), these light "films" became thicker. After 30\% chitosan added (Figure $1(\mathrm{~d})$ ), these films were so dense that the aerogel structure had less existing pores. This is because of the characteristic properties of chitosan, natively containing the high content of primary amino groups. Cellulose with several compounds which having primary amino groups, such as polyethylenimines, ethylenediamine, and benzyl amine, revealed the formation of new chemical bonds in the materials processed even at room temperature [20]. Here aerogel making employed the main reaction between amino and carbonyl groups, besides hydrogen bonds of cellulose and chitosan groups, to improve its mechanical strength [20, 25]. Since NFC has much more exposed carboxyl/carbonyl groups than MFC, theoretically $\mathrm{NFC/CH}$ aerogel would result in more reaction between NFC and chitosan. In Figure 1, we did observe the tighter structure of $\mathrm{NFC} / \mathrm{CH}$ aerogels (pictures E, F, and $\mathrm{G}$ in Figure 1) than that of $\mathrm{MFC} / \mathrm{CH}$ (pictures $\mathrm{A}, \mathrm{B}, \mathrm{C}$, and $\mathrm{D}$ in Figure 1). Fewer pores but more "films" were formed in NFC/CH aerogel. The data presented in Tables 1-3 are additional evidences to confirm this observation.

After Ag-NPs loaded, these aerogels exhibited similar SEM morphology to that of Ag-NPs unloaded, in which structures are denser with the increase in chitosan. This denser structure caused the nanoparticles to fill differently. Denser aerogel has relatively tight structure and fewer capillaries, which penetrates less Ag+ and reducing solution, resulting in fewer Ag-NPs generated. Table 3 shows the amount of Ag-NPs in MFC/NFC aerogels after $575^{\circ} \mathrm{C}$ 


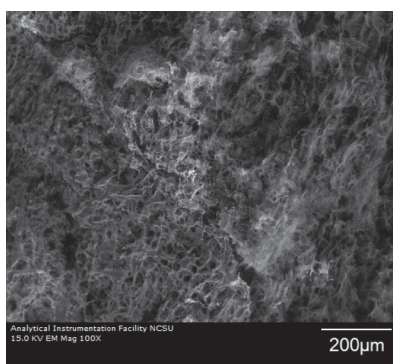

(a)

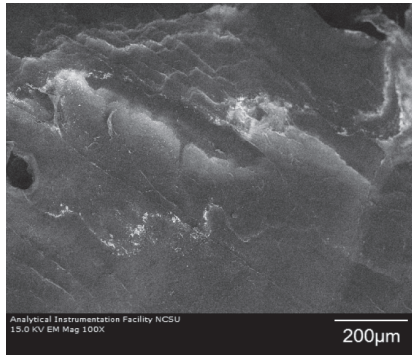

(d)

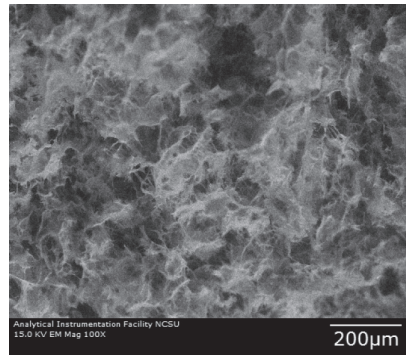

(b)

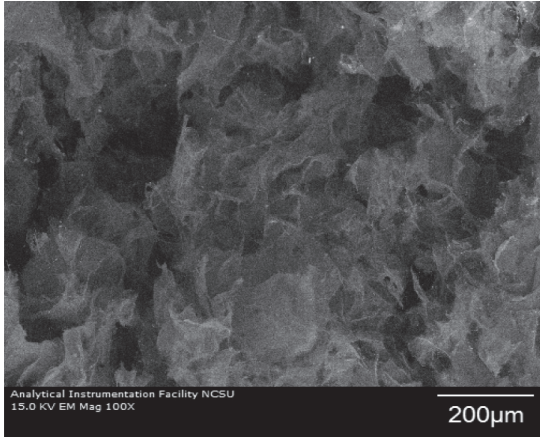

(e)

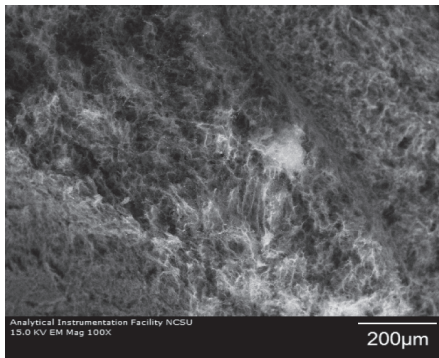

(c)

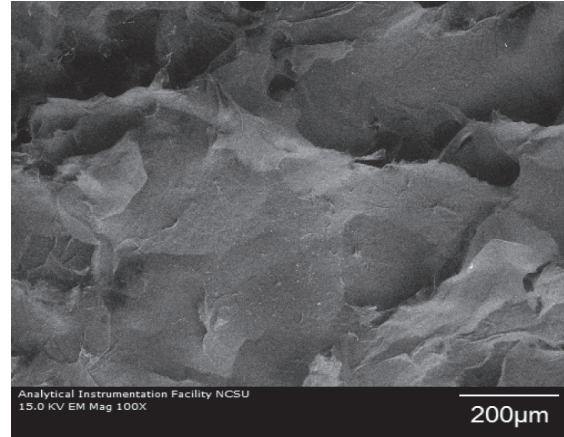

(f)

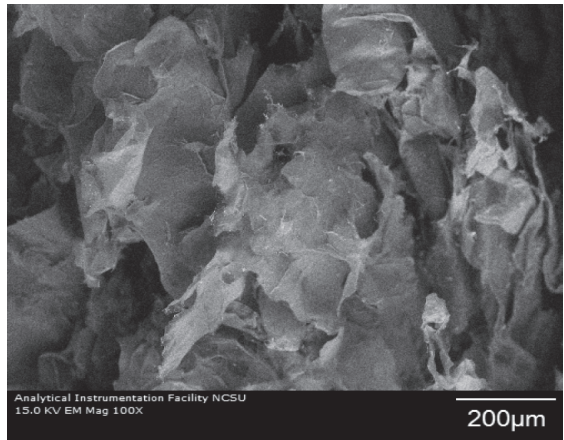

(g)

FiguRE 1: MFC/NFC aerogel SEM with different chitosan additions. MFC/CH ratio: (a) 100/0, (b) 90/10, (c) 80/20, and (d) 70/30; NFC/CH ratio: (e) $90 / 10$, (f) $80 / 20$, and (g) $70 / 30$.

TABLE 1: Data on the density of chitosan- (CH-) added MFC/NFC aerogel.

\begin{tabular}{lcccc}
\hline Aerogel density $\left(\mathrm{g} / \mathrm{cm}^{3}\right)$ & $\mathrm{CH} 0 \%$ & $\mathrm{CH} 10 \%$ & $\mathrm{CH} 20 \%$ & $\mathrm{CH} 30 \%$ \\
\hline $\mathrm{MFC} / \mathrm{CH}$ & 0.0258 & 0.0397 & 0.0425 & 0.0426 \\
$\mathrm{NFC} / \mathrm{CH}$ & - & 0.0399 & 0.0442 & 0.0451 \\
\hline
\end{tabular}

Note: pure MFC aerogel densities were obtained with $0.0258,0.0505$, and $0.0807 \mathrm{~g} / \mathrm{cm}^{3}$ from $2 \%, 5 \%$, and $8 \%$ MFC gel concentrations, respectively.

oxygen for $25 \mathrm{~min}$ isothermal time under TGA. It disclosed that NFC aerogel has loaded fewer Ag-NPs than MFC aerogels.

In order to investigate how the nanoparticles are located in these aerogels, the $\mathrm{MFC} / \mathrm{CH}$ and $\mathrm{NFC} / \mathrm{CH}$ aerogels with Ag-NPs were scanned by using SEM in high magnification. The images are shown in Figure 2. Since nanoparticles are expected to locate evenly in smaller size on the composite, any aggregates should be avoided. All NFC aerogels showed particle aggregates (pictures D, E, and Figure 2), but only
MFC-30\% $\mathrm{CH}$ aerogel showed Ag-NP aggregates (picture C in Figure 2). The formation of Ag-NP aggregates may be from the rich hydroxyl and carboxyl groups which absorb much $\mathrm{Ag}+$ solution. But there are less free hydroxyl and carboxyl groups in denser aerogel after drying. While 'films' are formed, the Ag+ concentrate was forced to sit in pores and then enwrapped as aggregates. These free groups present negative charge, providing the anchor sites for $\mathrm{Ag}+$ uniformly in loose aerogels of MFC/CH with $10 \%$ and $20 \%$ chitosan. They are better aerogels for nanoparticle loading.

Ag-NP size and distribution for aerogel are very important. Based on good particle distribution in SEM images shown in Figure 2, MFC aerogel with 20\% CH was chosen and TEM observation was conducted (see Figure 3). From the TEM image, we can see that the particles are monodispersed and have very small size. Using ImageJ software, the TEM image shown in Figure 3(a) was analyzed for particle size distribution automatically with every size scale bar $(0.72 \mathrm{~nm})$ starting at $4.13 \mathrm{~nm}$. Most particles are in the 
TABLE 2: BET of both MFC/CH aerogel- and NFC/CH aerogel-loaded Ag-NPs.

\begin{tabular}{|c|c|c|c|c|c|}
\hline Aerogel $\mathrm{BET} \mathrm{m}^{2} / \mathrm{g}$ & CH10\% Ag loaded & CH20\% Ag loaded & CH30\% Ag loaded & CH0\% Ag loaded & $\mathrm{CH} 30 \%$ with no $\mathrm{Ag}$ \\
\hline $\mathrm{MFC} / \mathrm{CH}$ & 13.4 & 11.3 & 8.8 & 13.2 & 9.7 \\
\hline $\mathrm{NFC} / \mathrm{CH}$ & 1.67 & 1.2 & 1.2 & - & - \\
\hline
\end{tabular}

TABLE 3: Ag-NP content in MFC/NFC aerogels.

\begin{tabular}{lcccc}
\hline Ag-NPs $\%$ & CH $0 \%$ & CH $10 \%$ & CH 20\% & CH 30\% \\
\hline MFC aerogel & 2.5 & 3.8 & 3.6 & 3.6 \\
NFC aerogel & - & 3.2 & 3 & 3 \\
\hline
\end{tabular}

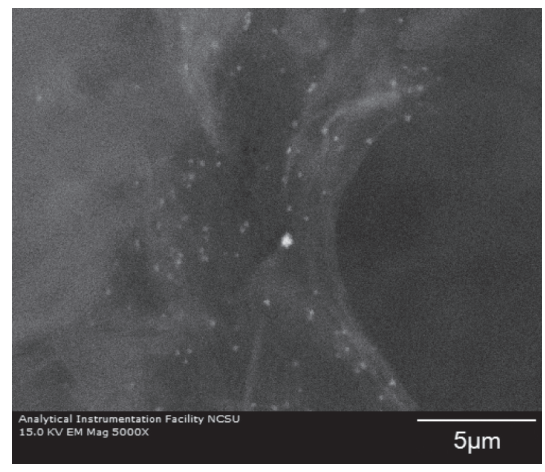

(a)

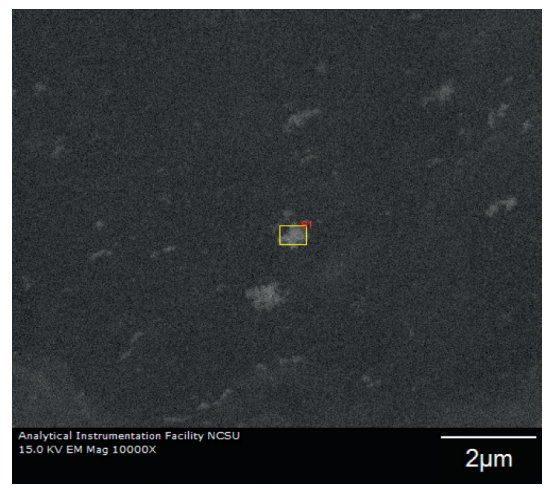

(d)

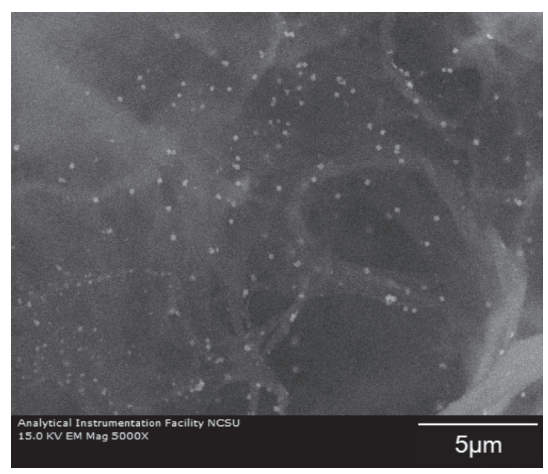

(b)

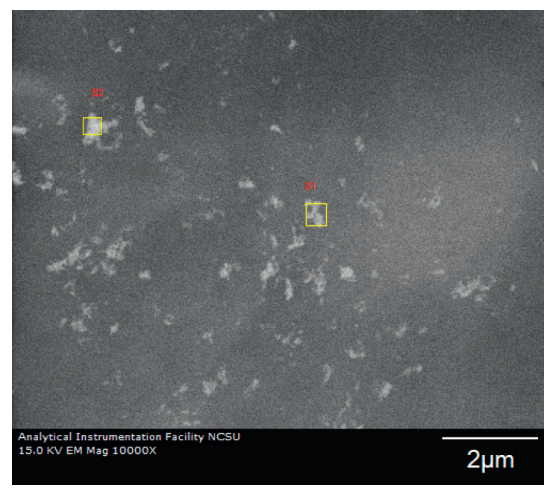

(e)

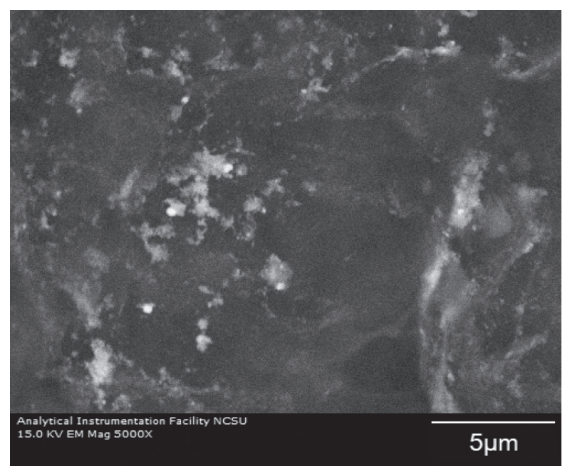

(c)

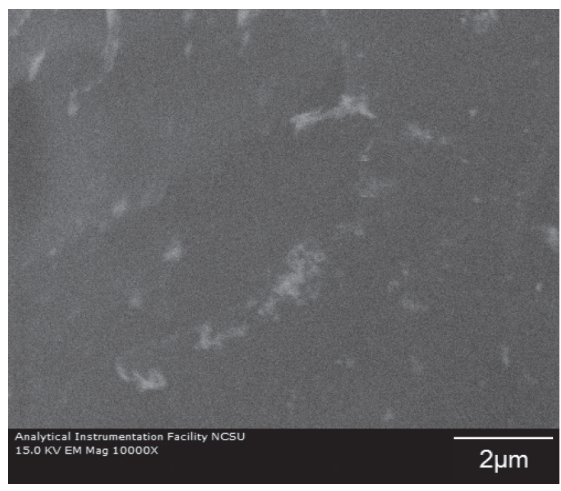

(f)

Figure 2: Ag-NPs in MFC/CH aerogel: (a) 10\%CH, (b) $20 \% \mathrm{CH}$, and (c) $30 \% \mathrm{CH}$; NFC/CH aerogel: (d) $10 \% \mathrm{CH}$, (e) $20 \% \mathrm{CH}$, and (f) $30 \% \mathrm{CH}$.

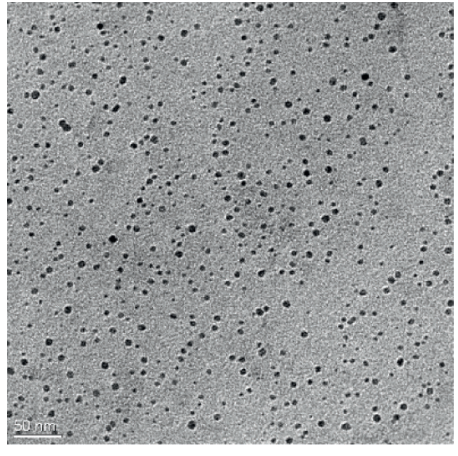

(a)

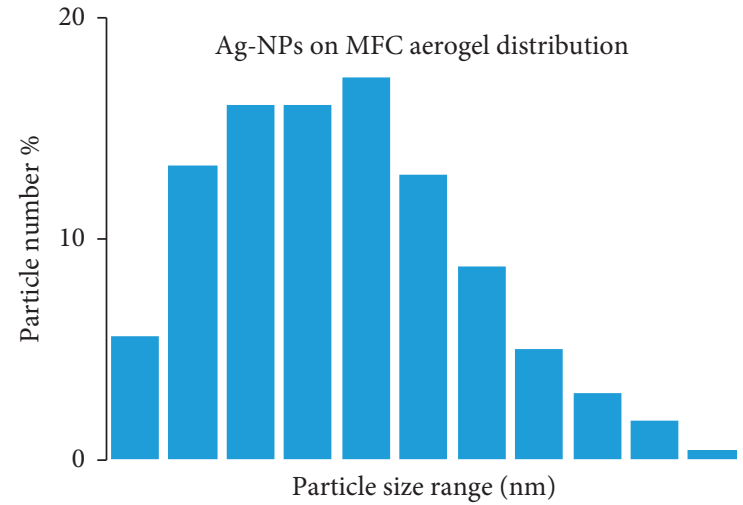

(b)

Figure 3: TEM images of MFC/CH aerogel-loaded Ag-NPs. 20\% CH (a) and particle size range (b). 


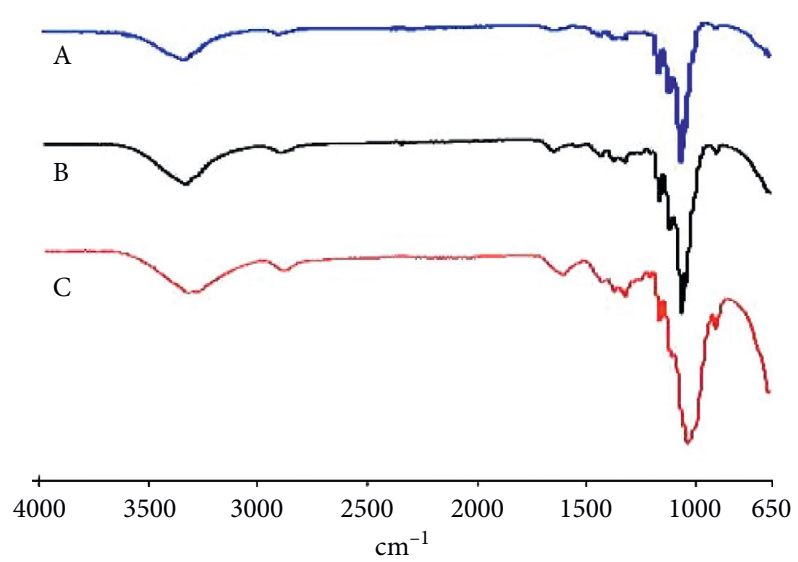

FIGURE 4: MFC/NFC aerogel FTIR spectra with chitosan 20\%. Note: A, MFC aerogel 0\% CH; B, MFC aerogel 20\% CH; C, NFC aerogel 20\% CH.

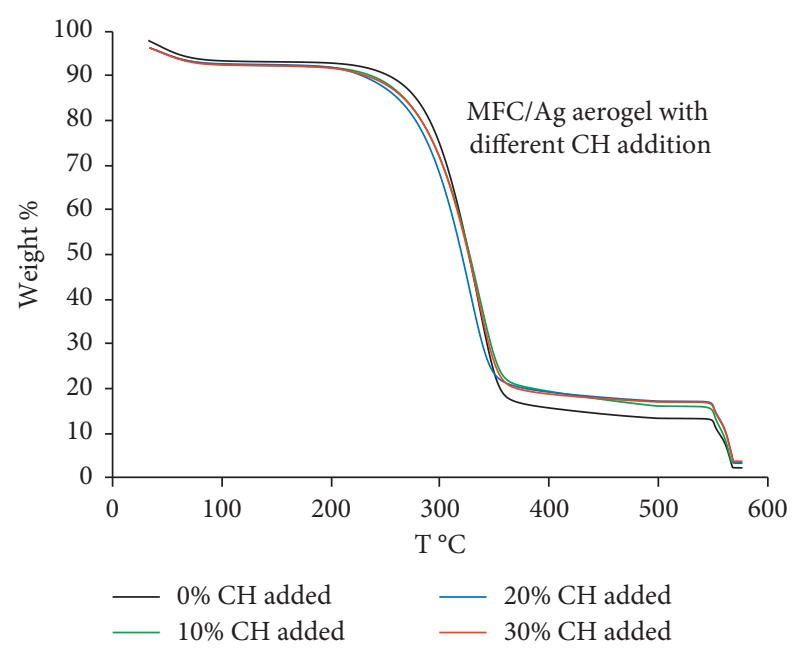

(a)

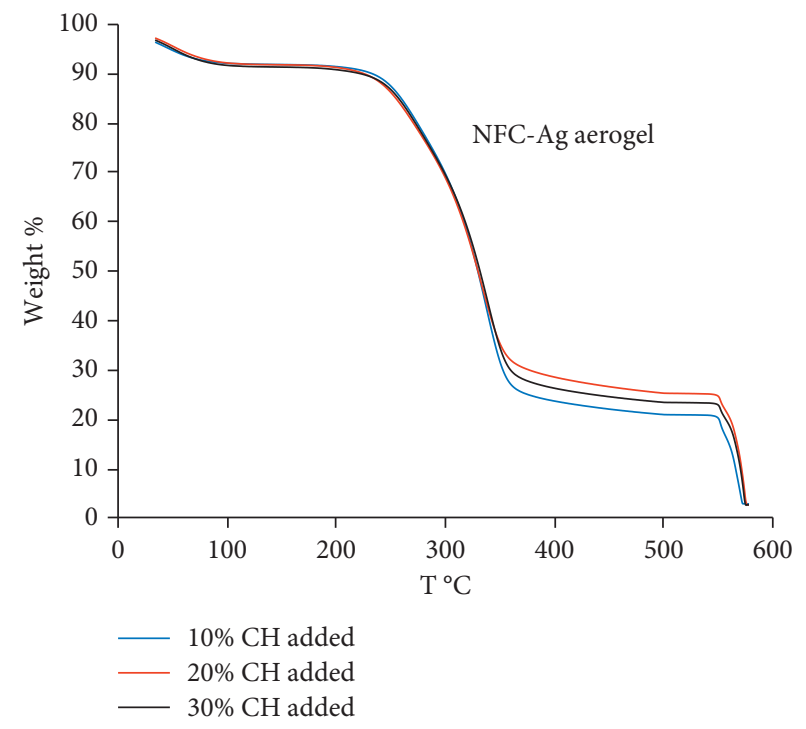

(b)

FIGURE 5: TGA behavior of MFC-Ag aerogels with different chitosan addition and NFC-Ag aerogels with different chitosan addition. Note: TGA under $\mathrm{N} 2$ atmosphere till $500^{\circ} \mathrm{C}$ and then switched to oxygen to $575^{\circ} \mathrm{C}$.

range of 4-9 $\mathrm{nm}$ with a mean diameter of $7.08 \mathrm{~nm}$. This is because the aerogel was treated by very dilute AgNO3 solution $(10 \mathrm{mM})$ and there were less $\mathrm{Ag}+$ surrounding nanofibrils, typically at negative charged groups, which are the anchors to be the nucleus of particle growth. Nanofibrils played an important role to physically stop the $\mathrm{Ag}+$ free movement, and then monodispersed small particles were formed. With more chitosan in aerogels, since chitosan amino groups consumed some carbonyl groups on MFC/ NFC, and most possibly chitosan molecules have strong hydrogen bonds with MFC/NFC (it is to observe "films" in SEM images), and these aerogels lost many uniform places to accommodate $\mathrm{Ag}+$, resulting in particle aggregates, as we can see in C, D, E, and F of Figure 2.

3.3. FTIR Spectroscopy. The spectra of MFC aerogel with $20 \%$ chitosan is shown in Figure 4, along with MFC aerogel blank and $\mathrm{NFC/CH}$ areogel (marked as $\mathrm{B}, \mathrm{A}$, and $\mathrm{C}$ respectively, in Figure 4). The peaks at $3330-3340 \mathrm{~cm}^{-1}$ corresponded to the stretching vibration of hydroxyl groups. These peak intensities reinforced for MFC/NFC aerogel blended chitosan due to the $\mathrm{N}-\mathrm{H}$ groups involvment [39]. The main change observed in the spectra is no sharp peaks at $1650 \mathrm{~cm}^{-1}$ which corresponded to the carbonyl stretch and $1560 \mathrm{~cm}^{-1}$ to the amino groups of chitosan in $\mathrm{B}$ and $\mathrm{C}$ of Figure 4, implying the chemical reaction between chitosan and cellulose [20]. This also explained why we observed the aerogel tight structure with chitosan under SEM.

3.4. Thermal Gravimetric Analysis (TGA). In view of the importance of thermal stability in many applications of MFC/NFC aerogels, we examined thermal decomposition of composite-loaded Ag-NPs by thermogravimetry (TGA, Perkin Elmer Q500, and heating rate of $10^{\circ} \mathrm{C} / \mathrm{min}$ ) in a nitrogen atmosphere under $500^{\circ} \mathrm{C}$, as shown in Figure 5. In all TGA curves, the small weight losses below $150^{\circ} \mathrm{C}$ 


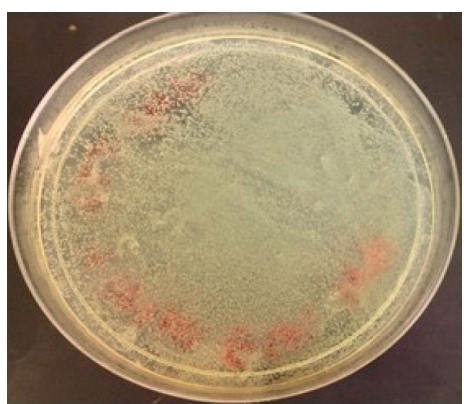

E. coli blank

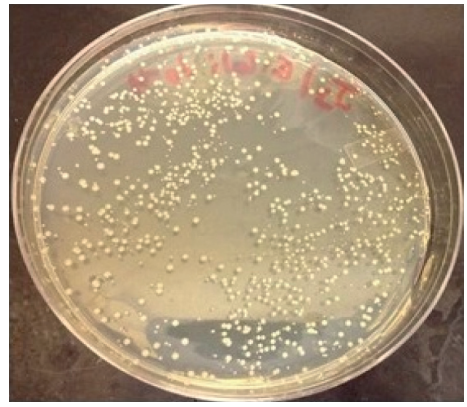

NFC-chitosan

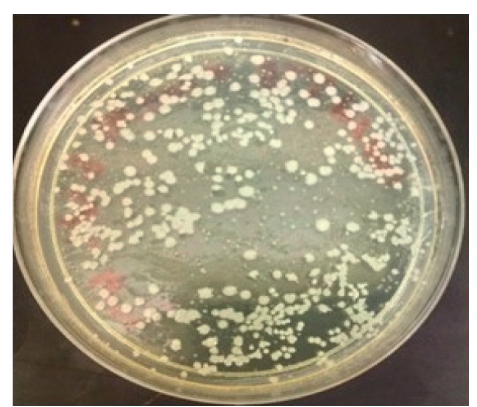

NFC-aerogel

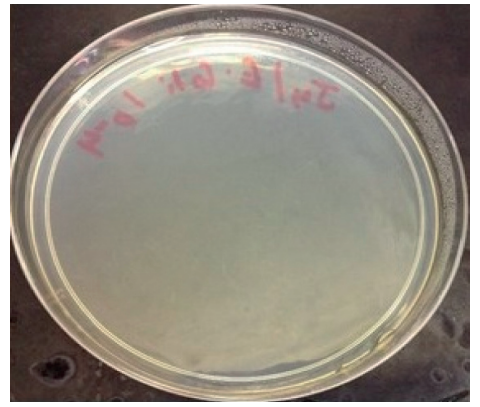

MFC-chitosan-AgNPs

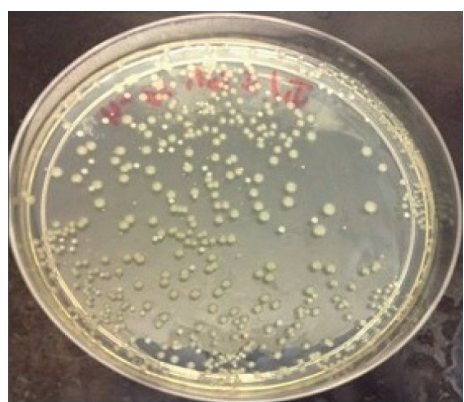

MFC-chitosan

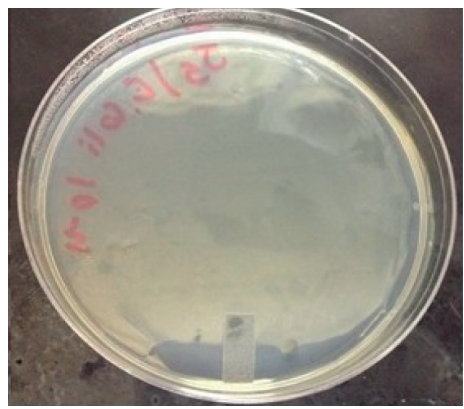

NFC-chitosan-AgNPs

Figure 6: Antibacterial activity of NFC, MFC/chitosan, NFC/chitosan, MFC/chitosan/Ag-NPs, and NFC/chitosan/Ag-NPs against E. coli.

apparently resulted from evaporation of adsorbed moisture. Under nitrogen, the decomposition behavior of the MFC-Ag aerogel was nearly the same with different levels of chitosan, the most weight loss taking place at $280^{\circ} \mathrm{C}$; meanwhile, NFC$\mathrm{Ag}$ aerogels have lower decomposition temperature of around $250^{\circ} \mathrm{C}$. Chitosan did not change both MFC and NFC aerogel thermal stability. In order to obtain the Ag-NP amount in aerogel, TGA under oxygen in the range of $500-575^{\circ} \mathrm{C}$ was continued. The isothermal time for $25 \mathrm{~min}$ at $575^{\circ} \mathrm{C}$ was set up. The results are listed in Table 3. The Ag$\mathrm{NPs}$ in $\mathrm{MFC} / \mathrm{CH}$ aerogel has slightly higher amount than that in $\mathrm{NFC/CH}$ aerogel, but the former has more uniform particle distribution.

3.5. Antibacterial Activity Testing. The antibacterial activity of these four samples MFC80/CH20, NFC80/CH20, MFC80/ $\mathrm{CH} 20 / \mathrm{Ag}-\mathrm{NPs}$, and NFC80/CH20/Ag-NPs was tested against E. coli by using the viable cell-counting method. NFC aerogel without chitosan was compared. The effects of aerogels on the growth of the recombinant bacteria E. coli are shown in Figure 6. "Blank" in Figure 6 means a plate which was produced from untreated bacterial solution. As shown in the plates, no bacterial colonies were observed at concentrations of $7 \times 107 \mathrm{CFU} / \mathrm{mL}$ for both $\mathrm{MFC} / \mathrm{CH}-\mathrm{Ag}-\mathrm{NPs}$ and $\mathrm{NFC} / \mathrm{CH}-$ Ag-NPs which represent the highest antibacterial activity. On the other hand, many bacterial colonies were observed for MFC-CH, NFC-CH, and NFC aerogels which implied poor antibacterial activity. Though chitosan is considered as a good natural polymer for medical application due to its antibacterial property, Ag-NPs in these aerogels imported much better inhibition of bacteria than chitosan. MFC/CH-Ag-NPs may have better antibacterial property than NFC/CH-Ag-NPs since the former has excellent nanoparticle distribution. This should be further examined.

\section{Conclusions}

In the current work, we successfully utilized chitosan as MFC/ NFC aerogel reinforcement biopolymer. The reinforced aerogels have overcome the water unstability. With more chitosan addition, MFC/NFC aerogels became denser which have tighter structures that are observed in SEM. Chemical reaction occured between chitosan and MFC/NFC. Comparing both $\mathrm{MFC} / \mathrm{CH}$ and $\mathrm{NFC} / \mathrm{CH}$ aerogels, $\mathrm{MFC} / \mathrm{CH}$ aerogel has higher BET surface area and lower density than $\mathrm{NFC/CH}$ aerogel. AgNPs were in site loaded to chitosan-added MFC/NFC aerogel. The MFC/CH aerogel performed much better than the NFC/ $\mathrm{CH}$ aerogel, which obtained monodispersed nanoparticles of Ag-NPs. Finally, both MFC/CH-Ag-NP and NFC/CH-Ag-NP aerogels exhibited excellent antibacterial activity. But the better antibacterial performance of both aerogels should be further examined.

\section{Data Availability}

The data used to support the findings of this study are available from the corresponding author upon request.

\section{Conflicts of Interest}

The authors declare no conflicts of interest.

\section{Acknowledgments}

The authors would like to thank Prof. Orlando J. Rojas and his group for their help during this research and in editing 
the manuscript. The Special Support Plan for Xijiang Innovation Team Plan 2016, High-Level Talent Cultivation of Guangdong Industry Polytechnic (No. KYRC2018-020), and Guangdong University Youth Innovation Fund Project (No. 2018GKQNCX032) are greatly acknowledged for the support.

\section{Supplementary Materials}

The SEM images showing the surface of MFC aerogel with $10 \%$ chitosan addition and 20\% chitosan addition. (Supplementary Materials)

\section{References}

[1] A. C. Pierre and G. M. Pajonk, "Chemistry of aerogels and their applications," Chemical Reviews, vol. 102, no. 11, pp. 4243-4266, 2002.

[2] U. Schubert, "Silica-based and transition metal-based inorganic-organic hybrid materials-a comparison," Journal of Sol-Gel Science and Technology, vol. 26, no. 1-3, pp. 47-55, 2003.

[3] E. Guilminot, F. Fischer, M. Chatenet et al., "Use of cellulosebased carbon aerogels as catalyst support for PEM fuel cell electrodes: electrochemical characterization," Journal of Power Sources, vol. 166, no. 1, pp. 104-111, 2007.

[4] R. Baetens, B. P. Jelle, and A. Gustavsen, "Aerogel insulation for building applications: a state-of-the-art review," Energy and Buildings, vol. 43, no. 4, pp. 761-769, 2011.

[5] J. Biener, M. Stadermann, M. Suss et al., "Advanced carbon aerogels for energy applications," Energy \& Environmental Science, vol. 4, no. 3, pp. 656-667, 2011.

[6] G. Nyström, A. Marais, E. Karabulut, L. Wågberg, Yi Cui, and M. M. Mahiar, "Self-assembled three-dimensional and compressible interdigitated thin-film supercapacitors and batteries," Nature Communications, vol. 6, no. 1, p. 7259, 2015.

[7] R. Gavillon and T. Budtova, "Aerocellulose: new highly porous cellulose prepared from cellulose- $\mathrm{NaOH}$ aqueous solutions," Biomacromolecules, vol. 9, no. 1, pp. 269-277, 2008.

[8] S. R. Ma, Q. Mi, J. Yu, J. He, and J. Zhang, "Aerogel materials based on cellulose," Progress in Chemistry, vol. 26, no. 5, pp. 796-809, 2014.

[9] H. Dong, J. F. Snyder, D. T. Tran, and J. L. Leadore, "Hydrogel, aerogel and film of cellulose nanofibrils functionalized with silver nanoparticles," Carbohydrate Polymers, vol. 95, no. 2, pp. 760-767, 2013.

[10] T. Nypelö, H. Pynnönen, M. Österberg, J. J. Paltakari, and J. Laine, "Interactions between inorganic nanoparticles and cellulose nanofibrils," Cellulose, vol. 19, no. 3, pp. 779-792, 2012.

[11] H. Valo, S. Arola, P. Laaksonen et al., "Drug release from nanoparticles embedded in four different nanofibrillar cellulose aerogels," European Journal of Pharmaceutical Sciences, vol. 50, no. 1, pp. 69-77, 2013.

[12] K. J. De France, T. Hoare, and E. D. Cranston, "Review of hydrogels and aerogels containing nanocellulose," Chemistry of Materials, vol. 29, no. 11, pp. 4609-4631, 2017.

[13] J. T. Korhonen, M. Kettunen, R. H. A. Ras, and O. Ikkala, "Hydrophobic nanocellulose aerogels as floating, sustainable, reusable, and recyclable oil absorbents," ACS Applied Materials \& Interfaces, vol. 3, no. 6, pp. 1813-1816, 2011.

[14] R. Sescousse, A. Smacchia, and T. Budtova, "Influence of lignin on cellulose- $\mathrm{NaOH}$-water mixtures properties and on aerocellulose morphology," Cellulose, vol. 17, no. 6, pp. 1137-1146, 2010.

[15] R. Sescousse, R. Gavillon, and T. Budtova, "Aerocellulose from cellulose-ionic liquid solutions: preparation, properties and comparison with cellulose- $\mathrm{NaOH}$ and cellulose-NMMO routes," Carbohydrate Polymers, vol. 83, no. 4, pp. 1766-1774, 2011.

[16] C. Aulin, J. Netrval, L. Wågberg, and T. Lindström, “Aerogels from nanofibrillated cellulose with tunable oleophobicity," Soft Matter, vol. 6, no. 14, pp. 3298-3305, 2010.

[17] H. Sehaqui, M. Salajková, Q. Zhou, and L. A. Berglund, "Mechanical performance tailoring of tough ultra-high porosity foams prepared from cellulose I nanofiber suspensions," Soft Matter, vol. 6, no. 8, pp. 1824-1832, 2010.

[18] R. J. Moon, A. Martini, J. Nairn, J. Simonsen, and J. Youngblood, "Cellulose nanomaterials review: structure, properties and nanocomposites," Chemical Society Reviews, vol. 40, no. 7, pp. 3941-3994, 2011.

[19] J. M. Urreaga and M. U. de la Orden, "Chemical interactions and yellowing in chitosan-treated cellulose," European Polymer Journal, vol. 42, no. 10, pp. 2606-2616, 2006.

[20] W. Zhang, Y. Zhang, C. Lu, and Y. Deng, "Aerogels from crosslinked cellulose nano/micro-fibrils and their fast shape recovery property in water," Journal of Materials Chemistry, vol. 22, no. 23, pp. 11642-11650, 2012.

[21] L. Guo, Z. Chen, S. Lyu, F. Fu, and S. Wang, "Highly flexible cross-linked cellulose nanofibril sponge-like aerogels with improved mechanical property and enhanced flame retardancy," Carbohydrate Polymers, vol. 179, pp. 333-340, 2018.

[22] A. Kumar, Y. Lee, D. Kim et al., "Effect of crosslinking functionality on microstructure, mechanical properties, and in vitro cytocompatibility of cellulose nanocrystals reinforced poly (vinyl alcohol)/sodium alginate hybrid scaffolds," International Journal of Biological Macromolecules, vol. 95, pp. 962-973, 2017.

[23] T. Nissilä, S. S. Karhula, S. Saarakkala, and K. Oksman, "Cellulose nanofiber aerogels impregnated with bio-based epoxy using vacuum infusion: structure, orientation and mechanical properties," Composites Science and Technology, vol. 155, pp. 64-71, 2018.

[24] G. Skjak-Brack, T. Anthonsen, P. Sandford et al., Chitin and Chitosan: Sources Chemistry, Biochemistry, Physical Properties, and Applications, Elsevier Applied Science, London, UK, 1989.

[25] Z. Li, L. Shao, W. Hu et al., "Excellent reusable chitosan/ cellulose aerogel as an oil and organic solvent absorbent," Carbohydrate Polymers, vol. 191, pp. 183-190, 2018.

[26] H. Yang, A. Sheikhi, and T. G. M. van de Ven, "Reusable green aerogels from cross-linked hairy nanocrystalline cellulose and modified chitosan for dye removal," Langmuir, vol. 32, no. 45, pp. 11771-11779, 2016.

[27] G. Meng, H. Peng, J. Wu et al., "Fabrication of superhydrophobic cellulose/chitosan composite aerogel for oil/ water separation," Fibers and Polymers, vol. 18, no. 4, pp. 706-712, 2017.

[28] Z. Liu, H. Wang, C. Liu et al., "Magnetic cellulose-chitosan hydrogels prepared from ionic liquids as reusable adsorbent for removal of heavy metal ions," Chemical Communications, vol. 48, no. 59, pp. 7350-7352, 2012.

[29] T. Zhai, Q. Zheng, Z. Cai, L.-S. Turng, H. Xia, and S. Gong, "Poly (vinyl alcohol)/cellulose nanofibril hybrid aerogels with an aligned microtubular porous structure and their composites with polydimethylsiloxane," ACS Applied Materials \& Interfaces, vol. 7, no. 13, pp. 7436-7444, 2015. 
[30] L. Wei, Q. Wu, Z Xin et al., "Enhanced thermal and mechanical properties of PVA composites formed with filamentous nanocellulose fibrils," Carbohydrate Polymers, vol. 113, pp. 403-410, 2014.

[31] Q. Zheng, Z. Cai, and S. Gong, "Green synthesis of polyvinyl alcohol (PVA)-cellulose nanofibril (CNF) hybrid aerogels and their use as superabsorbents," Journal of Materials Chemistry A, vol. 2, no. 9, pp. 3110-3118, 2014.

[32] I. S. Arvanitoyannis, A. Nakayama, and S.-I. Aiba, "Chitosan and gelatin based edible films: state diagrams, mechanical and permeation properties," Carbohydrate Polymers, vol. 37, no. 4, pp. 371-382, 1998.

[33] A. Sionkowska, M. Wisniewski, J. Skopinska, C. J. Kennedy, and T. J. Wess, "Molecular interactions in collagen and chitosan blends," Biomaterials, vol. 25, no. 5, pp. 795-801, 2004.

[34] Y. Zhou, S. Fu, L. Zhang, H. Zhan, and M. V. Levit, "Use of carboxylated cellulose nanofibrils-filled magnetic chitosan hydrogel beads as adsorbents for $\mathrm{Pb}(\mathrm{II})$, , Carbohydrate Polymers, vol. 101, pp. 75-82, 2014.

[35] M. S. Toivonen, S. Kurki-Suonio, F. H. Schacher, S. Rojas, O. J. Rojas, and O. Ikkala, "Water-resistant, transparent hybrid nanopaper by physical cross-linking with chitosan," Biomacromolecules, vol. 16, no. 3, pp. 1062-1071, 2015.

[36] T. Saito, S. Kimura, Y. Nishiyama, and A. Isogai, "Cellulose nanofibers prepared by TEMPO-mediated oxidation of native cellulose," Biomacromolecules, vol. 8, no. 8, pp. 2485-2491, 2007.

[37] A. E. Horvath and T. Lindström, "Indirect polyelectrolyte titration of cellulosic fibers-surface and bulk charges of cellulosic fibers," Nordic Pulp \& Paper Research Journal, vol. 22, no. 1, pp. 87-92, 2007.

[38] S. C. M. Fernandes, L. Oliveira, C. S. R. Freire et al., "Novel transparent nanocomposite films based on chitosan and bacterial cellulose," Green Chemistry, vol. 11, no. 12, pp. 2023-2029, 2009.

[39] S. Peng, Y. Liu, Z. Xue et al., "Modified nanoporous magnetic cellulose-chitosan microspheres for efficient removal of $\mathrm{Pb}(\mathrm{II})$ and methylene blue from aqueous solution," Cellulose, vol. 24, no. 11, pp. 4793-4806, 2017. 